\title{
8 Databionic Swarm (DBS)
}

This chapter introduces a new concept for the use of swarm intelligence. It makes use of insights from the previous chapter and proposes a projection method based on a swarm of intelligent agents called DataBots [Ultsch, 2000c]. This new swarm is called a polar swarm (Pswarm) because its agents move in polar coordinates based on symmetry considerations (see [Feynman et al., 2007, pp. 147-153, 745]). All parameters are automatically chosen according to, and directly based on, the appropriate high-dimensional definition of distance. The main idea of Pswarm is to combine the concepts of swarm intelligence and self-organization with non-cooperative game theory [Nash, 1950]. The main advance is the reliance on the concept of emergence [Ultsch, 2007] instead of the optimization of an objective function. This allows Pswarm to preserve structures in data sets that are characterized by discontinuity.

The extensive analysis of ant-based clustering (ABC) methods that has been performed in previous work allows the formulation of a precise mathematical definition of pheromonal stigmergy (a scent) [Herrmann/Ultsch, 2009]. The scent is defined in each neighborhood using an annealing scheme. The approach based on neighborhood reduction during the annealing process was invented by Kohonen [Kohonen, 1982b] and was used, for example, in [Demartines/Hérault, 1995; Hinton/Roweis, 2002; Ultsch, 1999]. In the context of swarm-based techniques, it was used for the first time in [Tsai et al., 2004]. Until now, finding the correct annealing scheme for a high-dimensional data set has remained a challenging task [Nybo et al., 2007]. The Pswarm algorithm utilizes randomness and the Nash equilibrium [Nash] of non-cooperative game theory to find an appropriate annealing scheme based on the data as given in the input space. For this purpose, the scent will be redefined as the payoff function ${ }^{51}$.

Having projected the high-dimensional points into two dimensions using Pswarm in section 8.1, the author applies the insights from chapters 4 and 5, particularly with regard to the generalized U-matrix, to propose a three-dimensional topographic map with hypsometric tints [Thrun et al., 2016a] based on the high-dimensional distances and the density of the two-dimensional projected points. Drawing further insights from [Lötsch/Ultsch, 2014], a semi-interactive, but parameter-insensitive, clustering approach is possible. The framework as a whole is called Databionic swarm (DBS) and has only two parameters: the number of clusters and the type of clustering (connected or compact). The key feature of DBS is that neither an overall objective function for the process nor the type of clusters sought is explicitly defined at any point during the Pswarm process. Both parameters can be deduced from a topographic map of the Pswarm projection and a dendrogram. For DBS clustering and Pswarm projection the CRAN R package Databionic swarm was used [Thrun, 2017].

\subsection{Projection with Pswarm}

This section introduces the Polar swarm (Pswarm algorithm, which is the key foundation for the clustering performed in the DBS framework. Although the entire algorithm is used in an interactive clustering approach, Pswarm by itself may be used as a projection method. Because

${ }^{51}$ However, DataBots will still be described as "smelling" their surroundings. 
this enables direct comparison with the swarm-organized projection (SOP) algorithm, Pswarm is introduced and discussed separately from DBS.

The analysis presented in the second section of this chapter strongly indicates that Pswarm outperforms SOP in terms of structure preservation by virtue of the property of emergence arising from its self-organizing collective behavior (see also chapter 10, section 3 ). In contrast to SOP and all other common projection methods [Venna/Kaski, 2007; Venna et al., 2010], Pswarm does not require any input parameters other than the data set of interest, in which case Euclidean distances are used in the input space. Alternatively, a user may also provide Pswarm with a matrix defined in terms of a particular dissimilarity measure, which is typically a distance but may also be a non-metric measure.

\subsubsection{Motivation: Game Theory}

The purpose of game theory is to model situations in which multiple players interact with each other and/or affect each other's outcomes [Nisan et al., 2007, p. 3]. The author of this thesis focuses on a general, not zero-sum, non-cooperative game of $n$ players [Neumann/Morgenstern, 1953 , p. 85] in which the choices each player makes determine the outcome for each player [Nisan et al., 2007, p. 9]. For this kind of game, Nash proved the existence of at least one equilibrium point [Nash, 1951]. The payoff for each player depends on not only his own choices but also the choices made by all other players [Nisan et al., 2007, p. 9]. Often, these choices are defined based on a set of mixed strategies for each player.

The key idea of Pswarm is to redefine a game as one annealing step (epoch), the players as DataBots, and the scent as a payoff function and to find an equilibrium for each game. In the context of Pswarm, the game consists of rules governing the movement of the DataBots, which is defined by the grid, the neighborhoods and the payoff function. Each DataBot searches for its strongest payoff by either moving across the grid or staying in its current position. A new game (epoch), which is defined based on the considered neighborhood radius $\mathrm{R}$, begins once an approximate equilibrium is achieved, i.e., once no movement of any DataBot leads to a stronger or better payoff for any other DataBot any longer (weak Nash equilibrium). This approach leads to a data-driven annealing scheme with steps which are not defined by parameters, contrary to SOP (e.g. threshold_max, i_max in Listing 7.1), CCA and ESOM (e.g. number of epochs) as well as NeRV ${ }^{52}$.

\subsubsection{Symmetry Considerations}

If we consider DataBots that occupy space in two dimensions, such as spheres or atoms, two points must be considered: first, no two DataBots are allowed to be in the same spot at the same time (collision avoidance), and second, a hexagonal lattice (tiling) is the densest possible packing of identical spheres in two dimensions [Hunklinger, 2009, p. 65]. Every such sphere represents a possible position for a DataBot. To ensure that the two-dimensional output space is used most efficiently, a hexagonal lattice tiling (grid) is used in Pswarm. To avoid problems associated with the surface of the grid, such as the positioning of DataBots near the border, the grid must have periodic boundary conditions and consequently must possess full translational symmetry [Haug/Koch, 2004, p. 34]. If the third dimension (e.g., as in a crystal) is disregarded, this two-dimensional grid can be represented by a three-dimensional torus [Pasquier, 1987], which

\footnotetext{
52 e.g. iterations, cg_steps, cg_steps_final in [Nybo/Venna, 2015, Thrun et al., 2017].
} 
is hereafter referred to as a toroidal grid. This means that the borders of the grid are cyclically connected. The periodicity of the grid is defined by its size in terms of the numbers of lines $L$ and columns $C$. If the grid were planar (not toroidal), undesired boundary effects could affect the outcome of any method.

Boundary effects are effects related to the borders of the output space in which the patterns of interactions across the borders of the bounded region are ignored or distorted, giving rise to shape effects, such that the shape imposed on the planar output space affects the perceived interactions between phenomena (see [McDonnell, 1995]). For example, if the output space is planar, it is unknown whether a projected point on the left border is similar (or dissimilar, in this case) to a projected point on the right border. It could be that the projection method is constrained to split similar points (with regard to the input space) in the output space. Another example is the distorted interactions between DataBots on the four borders when the output space is planar. Compared with a planar output space, a toroidal output space imposes fewer constraints on a projection (or clustering) $\operatorname{method}^{53}$ and therefore enables a more optimized folding of the high-dimensional input space. A toroidal output space (in the case of Pswarm, a grid) possesses the advantage of translational symmetry in two dimensions, and in this case, the direction of a DataBot's movement is less important than its extent (length) because of the periodicity (of the grid).

In addition to the above considerations, the positions on the grid are coded using polar coordinates because the distances between DataBots on the grid will be most important in later computations of the neighborhoods and the annealing scheme. Consequently, based on the relevant symmetry considerations, a transformation of the Cartesian $(x, y)$ coordinate system into polar coordinates $(r, \phi) \in O$ is proposed as follows:

$$
\begin{aligned}
& r=x^{2}+y^{2} \\
& \phi=\tan ^{-1}\left(\frac{y}{x}\right) * \frac{180}{\pi}
\end{aligned}
$$

Hereafter, $r$ represents the length of a DataBot's movement (jump), and $\phi$ represents the direction of that movement.

Previously, the size of any grid (e.g., in SOP or emergent self-organizing map (ESOM)), as defined by the numbers of lines $L$ and columns $C$, had to be chosen by the user. Choosing an incorrect size could result in a poor projection of the data. This was noted in previous works describing DataBot approaches prior to the development of the SOP algorithm [Kohlhof, 2010]. By contrast, in Pswarm, the grid size is chosen automatically, subject to three conditions. Let $\widetilde{D}$ be an upper triangle of the matrix of the input distances, let $N$ be the number of DataBots, let $\alpha$ be the number of possible jump positions, let $\beta \in(0.5,1]$ be a scaling factor, and let p $_{99}$ and p01 denote the 99-th and first percentiles, respectively, of the distances; then, the conditions for determining the grid size are

$$
\begin{aligned}
& \frac{\sqrt{C^{2}+L^{2}}}{1} \geq \frac{p_{99}(\widetilde{D})}{p_{01}(\widetilde{D})}=: A \\
& L * C \geq \alpha * N \quad
\end{aligned}
$$

\footnotetext{
${ }^{53}$ To the author's knowledge, only the emergent self-organizing map (ESOM) and the swarm-organized projection (SOP) method offer the option to switch between planar and toroidal spaces (see [Ultsch, 1999], [Herrmann, 2011, p. 98]).
} 


$$
\frac{L}{C}=\frac{\beta}{1}
$$

These conditions result in the following bi-quadratic equation:

$$
\begin{gathered}
C^{4}-A^{2} * C^{2}+\alpha^{2} * N^{2}=0 \\
Z_{1 / 2}=A^{2} \pm \frac{1}{2} \sqrt{A^{4}-\frac{\alpha^{2}}{4} N^{2}} \\
=C=\left\{\begin{array}{c}
\frac{1}{\sqrt{2}} \sqrt{A^{2}+\sqrt{A^{4}-\frac{\alpha^{2}}{4} N^{2}},} A^{4} \geq \frac{\alpha^{2}}{4} N^{2} \\
\text { approximation, } A<\frac{\alpha^{2}}{4} N^{2}
\end{array}\right.
\end{gathered}
$$

The first condition ensures that the shortest and longest distances of interest are assignable to grid units. It defines the possible resolution of high-dimensional structures in the grid. The second condition ensures that there are sufficient available positions to which a DataBot can jump. The third condition causes the grid to be more rectangular than square because in the case of SOMs, "rectangular maps outperform square maps" [Ultsch/Herrmann, 2005]. The first two conditions are used to formulate the bi-quadratic equation under the assumption of equality (see Eq. 8.4). If the equation has no solution for the case of $A^{4}<\frac{\alpha^{2}}{4} N^{2}$, then conditions I and III are used to generate approximate solutions. The scaling factor $\beta$ is arbitrary and used only to ensure a solution in the case of approximation but it is not a parameter which has to be chosen. In this solution space, a solution that fulfills condition II is chosen.

\subsubsection{Algorithm}

Several previously developed ideas are applied in Pswarm: scent ${ }^{54}$ [Herrmann/ Ultsch, 2008a], DataBots [Ultsch, 2000c] and the decreasing neighborhood radius proposed for DataBots by [Kämpf/Ultsch, 2006]. The decrease in the radius is based on the data and is not predefined by parameters, which was a goal of [Herrmann, 2011], where it was called selfadaptation. The underlying idea of the decreasing radius approach is to promote self-organization, first of a global structure and then of local structures [Kämpf/Ultsch, 2006].

The intelligent agents of Pswarm operate on a toroidal grid where the positions are coded using polar coordinates, $i_{\phi}(r) \in O$. This permits the DataBots' movement, the neighborhood function and the annealing scheme to be precisely defined. The numeric vector $z_{j}$ associated with each DataBot $b_{j}$ represents its distances from all other DataBots in the input space I. The outputspace distances are coded using only the polar coordinate $r$. The size of the squared-distance matrix D is defined by the number of DataBots.

After the assignment of initial random positions on the grid $\mathrm{O}$ (and therefore random output distances) to the DataBots in Listing 8.1, a data-driven decreasing of the radius $\mathrm{R}$ begins. In every iteration, a portion of the DataBots are allowed to jump if the payoff in one of their new positions is better (stronger) than that in their old positions. In other words, each DataBot is given a chance $c(R)$ to try new positions on the grid.

The chance $c(R): \mathbb{N} \rightarrow>[0.05,0.5]$ is a continuous, monotonically decreasing linear function addressing the number of the DataBots which are allowed to search for a new position to jump

\footnotetext{
${ }^{54}$ Called topographic stress in [Herrmann/Ultsch, 2008].
} 
to. Initially, many ${ }^{55}$ DataBots are allowed to jump simultaneously to reproduce the coarse structure of the high-dimensional data set. However, as the algorithm progresses to address finer structures, only a small number ${ }^{56}$ of DataBots may move simultaneously. The chance function depends on the number of DataBots and on the current radius $R$ and consequently is based on the data itself.

In Pswarm, the length of a possible DataBot jump is not reduced during annealing ${ }^{57}$. The possible jumps of DataBots to new positions are drawn from a uniform distribution; therefore, the probability of selection is the same for all possible jumps, from a jump to zero to a jump to $R_{\max }$ in any direction. The direction of a jump to a new position is chosen separately from among all positions corresponding to an equal jump length. This approach prevents local minima from causing the DataBots to become stuck in an incorrect cluster because the length of their jump is smaller than half of the cluster's diameter. No DataBot is allowed to jump to an occupied position. Each DataBot may choose one of the four best different positions $(\alpha=4)$ in different directions to which to jump if it is sampled for jumping. This approach ensures a high probability that every sampled DataBot will find a free position.

function Positions $O=P$ Swarm(matrix $D(l, j)$ )

for all $z_{i} \in I$ : assign an initial random polar position $i_{\phi}(r) \in O$ on the grid

to generate $N$ DataBots $b_{i} \in B$

for $R=\{$ Rmax $=$ Lines $/ 2, \ldots, R \min \}$ do

calculate chance $c(R)$

Repeat for each iteration

$$
\begin{aligned}
& c=\operatorname{sample}(c(R), B) \\
& m_{k}(c)=\text { uniform }(1, \text { Rmax }), \text { with } k=1, \ldots, \alpha, m_{k}(c) \in O \\
& l(c)=\underset{j \in\left\{i, m_{k}(c)\right\}}{\operatorname{argmax}}\left(\lambda\left(b_{j}, R\right)\right) \\
& l(! c)=i \\
& S=\sum_{l=1}^{N} \lambda_{l}\left(b_{l}, R\right)
\end{aligned}
$$

$$
\text { Until } \frac{\partial S(e, \lambda(R))}{\partial e}=0
$$

return $O$ in Cartesian coordinates

end function Pswarm

Listing 8.1: The Pswarm algorithm consisting of $N$ DataBots. New possible positions are depicted with $m_{k(i)}(c)$ where $\mathrm{k}$ indicates up to the number of $\alpha$ polar positions $i_{\phi}(r)$ chosen with an equal chance in the range from 1 up to Rmax (uniform) relative to the old position $i$ and the old position with $i$ of a DataBot which has a chance $c$ to jump. After the decision to jump or not to jump the position is depicted with 1(c). All other DataBots do not search for a new position depicted with $! c$ and remain on their old position $i$. The data-driven annealing scheme (repeat/until) is parameter free due to the application of the Nash equilibrium of game theory (see 8.1.6).

\footnotetext{
${ }^{55}$ However, no more than half of the DataBots are allowed to search for a new position.

56 At the end exactly five percent of all DataBots.

${ }^{57}$ Unlike in the SOP algorithm.
} 


\subsubsection{Data-driven Annealing Scheme}

Let each annealing step be defined as an epoch $e$; then, a new epoch begins (and a game ${ }^{58}$ ends) if the radius $R$ is reduced by the condition defined below.

Let $r(j, l)$ be the one-dimensional distance from $1 \in O$ to $\mathrm{j} \in O$ in polar coordinates $(r, \phi)$ as specified by the radius $R_{e}$; then, the neighborhood function "Cone" is defined as

$$
h_{R}=\left\{\begin{array}{c}
h_{R}: R_{e} \rightarrow[0,1]: \\
1-\frac{r(j, l)^{2}}{\pi R_{e}^{2}}, \text { iff } \frac{r(j, l)^{2}}{\pi R_{e}^{2}}<1 \\
0, \text { otherwise }
\end{array}\right.
$$

where $R_{e}$ is the radius of the neighborhood during epoch $e$.

Let $D(l, j)$ be the distance between $\mathrm{x}_{\mathrm{l}}, \mathrm{x}_{\mathrm{j}} \in \mathrm{I}$, and let $r(j, l)$ be the one-dimensional radial distance in two-dimensional polar coordinates $(r, \varphi)$ in the output space $\mathrm{O}$; then, in Pswarm, the scent around a DataBot $b_{j}$ is redefined to

$$
\lambda_{e}\left(b_{j}, R_{e}, S_{0}\right)=\left\{\begin{array}{cl}
S_{0}-\frac{\sum_{\mathrm{l} \in I} h_{R}(r(j, l)) * D(j, l)}{\sum_{\mathrm{l} \in I} h_{R}(r(j, l))}, & \text { iff } \sum_{1 \in W} h_{R}(r(j, l))>0 \\
S_{0}, & \text { otherwise }
\end{array}\right.
$$

where

$$
S_{0}=\sum_{j}\left|\lambda\left(b_{j}, R_{\max }, 0\right)\right|
$$

Following the discussion in section 8.1.2, the scent $\lambda\left(b_{j}, R\right)$ is identified as the payoff function $\lambda_{e}\left(b_{j}, R\right): \mathbb{R}_{0}^{+} \times O \rightarrow \mathbb{R}_{0}^{+}$for a DataBot.

The high-dimensional input distances $D(l, j)$ must be calculated only once, which is done prior to starting the algorithm, thereby reducing the computational cost. The computational cost of the algorithm does not depend on the dimension of the data set but does depend on the number of DataBots and the number of possible jump positions $\alpha$. Additionally, Pswarm allows the conversion of distances or dissimilarities into two-dimensional points.

Let $e$ be the current epoch, let $R_{e}$ be the current neighborhood radius, and let $b_{j} \in B$ denote the DataBots; then, the sum of all payoffs is the current global happiness, which may be called the stress ${ }^{59} S\left(e, R_{e}\right)$, and is defined as

$$
S\left(e, R_{e}\right)=\sum_{j} \lambda_{e}\left(b_{j}, R_{e}\right)
$$

The neighborhood is reduced if the derivative of the current global happiness is equal to zero:

$$
\frac{\partial S\left(e, R_{e}\right)}{\partial e}=0
$$

which is called the equilibrium of happiness condition. The neighborhood radius $\mathrm{R}$ is reduced from $R_{\max }$ toward $R_{\min }$ with a step size of 1 if the derivative of the sum of all payoffs $\lambda_{e}$ is equal to zero. This is the case if a (weak) equilibrium for all DataBots is found.

Because not all DataBots are allowed to jump simultaneously during a single iteration, as imposed by the function sample $(c(R), B)$, the DataBots are able to pay off their neighborhoods

\footnotetext{
${ }^{58}$ In the context of game theory.

59 To simplify the comparison with SOP.
} 
more often, thereby promoting the process of self-organization. By searching for an equilibrium, the net number of DataBots that would like to jump or are unhappy is irrelevant to the self-adaptive annealing process. Instead, the decision to shrink the neighborhood size or to proceed to the next epoch $e$ is made based on a Nash equilibrium [Nash, 1950]. The criterion is clearly defined to correspond to the condition in which the global amount of happiness in the current epoch remains unchanged, which is defined as the equilibrium of happiness, $\frac{\partial S}{\partial e}=0$.

\subsubsection{Annealing Interval}

Rmax is equal to Lines/2 if Lines $<$ Columns to prevent self-interaction of the DataBots. If the radius $\mathrm{R}$ were to be greater than Lines/2, then the neighborhood of a given DataBot would overlap with itself because of the toroidal nature of the grid. Moreover, the probability density function for choosing a new position cannot be uniformly (or Gaussian) distributed in this case because border positions can be reached from two directions $\phi$ on a toroidal grid.

Rmin is determined by the size of the grid and the number of DataBots. It is set to a value that allows every DataBot to smell a minimum of $5 \%$ of the other DataBots if they are distributed uniformly ${ }^{60}$. This selection is inspired by an emergent phenomenon called an ant mill [Schneirla, 1971, pp. 281-283]: Army ants are an aggressive, nomadic species, incessantly moving around. Based on its payoff, every ant follows another ant in front of it. If the head of the ant colony runs into the tail of the colony, the ants form a so-called circle of death, because they keep moving until they die. This phenomenon would not occur if the ants were able to smell a region farther ahead of them.

\subsubsection{Convergence}

In game theory, for a game with egoistic agents, a solution concept exists called the Nash equilibrium [Nash, 1950].

Let $(P, \Lambda)$ be a game with $\mathrm{n}$ DataBots $b_{i}, \mathrm{i}=1, \ldots, N$, where $P$ is a set of movement strategies and $\Lambda=\left\{\lambda_{\mathrm{e}, \mathrm{i}}\left(b_{i}, R_{e}=\mathrm{cons} t\right) \mid i=1, \ldots, N\right\}$ is the payoff function evaluated for every grid position $w_{i} \in P_{i}$. Each DataBot chooses a movement strategy consisting of a probability associated with a position on the grid. Upon deciding on a position, a DataBot receives the payoff defined by the scent. $P$ is a set of mixed strategies that are chosen stochastically with fixed probability in the context of game theory. Nash proved that in this case, the following equilibrium exists:

$$
\forall i . w_{i}, b_{i} \in P: \lambda_{i}\left(b_{i}{ }^{\prime}\right) \geq \lambda_{i}\left(b_{i}\right)
$$

The strategy $b_{i}$ is the equilibrium, for which no deviation in strategy (position on the grid) by any single DataBot results in a greater profit for that DataBot. In the case of Pswarm, the Nash equilibrium is called weak because there may be more than one strategy with the same payoff for some DataBots. Because of the existence of this equilibrium, the Pswarm algorithm will always converge.

\footnotetext{
${ }^{60} \mathrm{Rmin}$ (and Rmax) are chosen automatically by the Pswarm algorithm based on the gird size and consequently based on the data.
} 


\subsection{Comparing Pswarm with a Previously Developed Approach}

Although the entire algorithm is used in an interactive clustering approach that does not require any sensitive input parameters, in this section, Pswarm is treated as an independent projection method and is compared with swarm-organized projection (SOP, see also chapter 10, section $3)$.

It will be demonstrated that changing the coordinate system from Cartesian to polar coordinates enables precise and practical definitions of neighborhoods, stigmergy and distances in the output space. With this approach, by using the Nash equilibrium [Nash, 1950] and modifying the DataBots' movements, it is possible to deduce a parameter-free and data-driven annealing scheme. This section will show that the self-adaptive annealing scheme of SOP requires important parameters and is, in fact, not always self-adaptive, as opposed to the Pswarm algorithm.

\subsubsection{Neighborhood Definition}

The main problem with regard to SOP lies in the neighborhood definition and annealing scheme of [Ultsch/Herrmann, 2010] and [Herrmann, 2011], as shown in Figure 8.1.

Because the lattice tiling is rectangular (quad grid), as is justified for Cartesian coordinates by [Ultsch/Herrmann, 2005], the neighborhoods are square and not round; this was explicitly defined in [Herrmann, 2011, p. 46] and remains unchanged in the SOP algorithm [Herrmann, 2011 , pp. 64-70], and it is relevant to the scent $\lambda$ (as defined in chapter 7.1 in Eq. 7.1).

In SOP, the following applies $d_{1}(l, j)=d_{2}(l, j)$, where these distances denote the lengths of jumps between $l, j=x, y$ in Cartesian coordinates. This means that the probability of selecting a diagonal position for a DataBot jump is equal to that of selecting a horizontal/vertical position in the SOP lattice because the two-dimensional Gaussian neighborhood consists of two Gaussian functions, from which the vertical and horizontal coordinates are drawn separately to determine the chosen lattice positions: $N(m(x), s=\sigma=R)+N(m(y), s=\sigma=R)$.

For the choice of new positions for the DataBots, Hermann proposed that the selection probability should a Gaussian [Herrmann, 2011, p. 64], where the center is the current position of the DataBot, $\mathrm{m}(\mathrm{x}, \mathrm{y})$, and the standard deviation s [Ultsch/Herrmann, 2010, p. 3] is equal to the radius R. In [Ultsch/Herrmann, 2010], a two-dimensional Gaussian distribution $N^{2}(m, s)$ was mentioned, but a practical solution to the problem of how to implement a two-dimensional Gaussian distribution on a discrete lattice was not addressed [Herrmann, 2011]. Moreover, the neighborhood considered in [Herrmann, 2011, p. 64] was defined only on a finite lattice. 


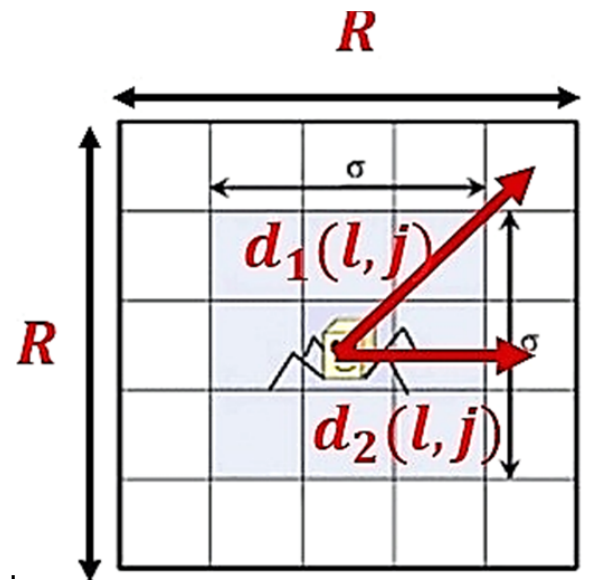

Figure 8.1: Neighborhood definition in the (rectangular) lattice tiling of a square shape of the SOP algorithm, adapted from [Herrmann, 2011, p. 47]. All positions defined at distances of less than or equal to $\mathrm{r}=2$ are shown. Independent of the coordinate system, the SOP lattice is rectangular, with a size of $(L, C)$.

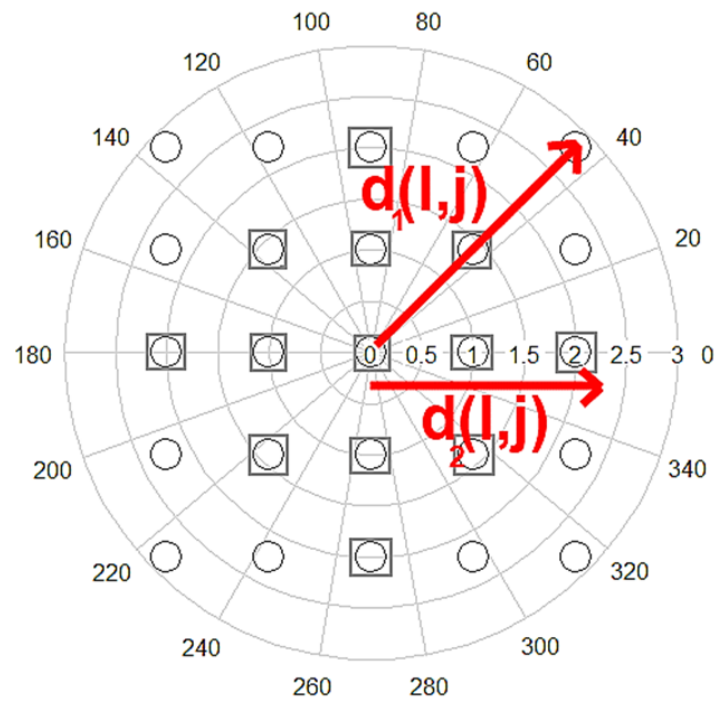

Figure 8.2: A similar rectangular lattice tiling of a square shape in polar coordinates for comparison ${ }^{61}$. In Pswarm, it applies $d_{1}(l, j) \neq d_{2}(l, j)$ for $\mathrm{j}, \mathrm{l}=\mathrm{r}, \phi$ in polar coordinates. All positions at distances smaller than or equal to $r=2$ are marked by gray squares. In this case, the neighborhood (Eq. 8.5) depends on a precise one-dimensional grid distance, and for Gaussian neighborhoods, jump positions can be drawn from $N(m(r), s=R)$. Independent of the coordinate system, the Pswarm (hexagonal) grid has a rectangular shape of borders, with a size of $(L, C)$.

On a toroidal grid or lattice (tiling), such a neighborhood will always overlap itself because Gaussian functions are never equal to zero. No solution for the case of a toroidal lattice was offered in [Herrmann, 2011]. Instead, in practice, the choice of a new DataBot position in the SOP algorithm is made by drawing separately from one normal distribution for the $\mathrm{x}$ coordinate

\footnotetext{
${ }^{61}$ In reality, Pswarm uses a hexagonal tiling instead of a rectangular tiling referenced as a grid.
} 
and another normal distribution for the y coordinate, where the means are the corresponding coordinates of the current position and the standard deviations are equal to the radius ${ }^{62} R$. However, the following inequality applies:

$$
N(m(x), s)+N(m(y), s) \neq N^{2}(m(x, y), s)
$$

Consequently, diagonal jumps are equal in length to horizontal and vertical jumps. However, [Bauer et al., 1999] argues that in a rectangular lattice, diagonal neighbors cannot be regarded as nearest neighbors. Moreover, the Gaussians overlap at the origin.

Based on symmetry considerations, a transformation from the Cartesian ( $\mathrm{x}, \mathrm{y})$ coordinate system to the polar $(r, \phi)$ coordinate system is exploited in Pswarm.

This allows Pswarm to use a more precise neighborhood definition with sharp borders in Eq. 8.5, as illustrated in Figure 8.2, and makes the calculation of Euclidean distances in the twodimensional output space unnecessary ${ }^{63}$. The neighborhood is defined only by the radius $r$ of the polar coordinates. If the radius exceeds the borders of the toroidal grid, then the distance and jump length can be adapted using a modulus operation if drawn from uniform distributions. Allowing the maximum possible jump lengths prevents the algorithm from becoming trapped in local minima: if the jump length is too short, there is a possibility that the DataBots may be unhappy in their positions but unable to find new positions because no open positions exist.

In contrast to Pswarm, in SOP, the neighborhood definition for the scent $\lambda$ remains vague. In [Herrmann, 2011, p. 63], it is stated that the development of SOP led to the revision of the ABC method based on Figure 8.1, where quadratic neighborhoods are explicitly defined [Herrmann, 2011, p. 46]. Still, this definition remained unchanged [Herrmann, 2011, pp. 64-70]. However, if the maximal radius is set to $\mathrm{R}>$ Lines/ 2 for Lines $<$ Columns, then the Gaussian function $F_{R}$ required to calculate the scent $\lambda$ [Herrmann, 2011, p. 64] overlaps itself if no sharp borders are defined or if the grid or lattice is not finite (see chapter 7.1 Eq. 7.1). This overlap changes the weights of the output-space distances and the probabilities of choosing new positions to which to jump.

Additionally, the neighborhood of the lattice in which the DataBot is moving is defined by equal (square) diagonal and vertical jumps, but the two-dimensional distances on the lattice are defined as Euclidean distances (radial). These definitions are inconsistent with each other. Thus, the annealing scheme of the SOP algorithm is more square (jump length, position probability) than radial (output-space Euclidean distance). In summary, the use of Gaussian functions prevents the possibility of precisely defining the DataBot jump length and neighborhood, and worse, the jump length and neighborhood are not consistent with the output distances; see Figure 8.1 .

More importantly, the radius $R$ does not define a border for the SOP neighborhood; instead, it defines only the standard deviation of the density of a normal distribution. This results in very large neighborhoods without sharp borders. The adaptation of this neighborhood definition for a toroidal lattice was not addressed, and if the definitions of [Herrmann, 2011] were to be used on a toroidal lattice without modification, this would lead to significant mistakes.

Consequently, the definition of the scent $\lambda$ is not consistent because the Euclidean output-space distance definition is inconsistent with the neighborhood definition.

${ }^{62}$ Taken from [Kohlhof, 2010] and Lutz Herrmann's 2011 Java implementation.

${ }^{63}$ A spherical coordinate system is the appropriate extension for a three-dimensional system. 
Only a polar coordinate approach, such as that used in Pswarm, allows the selection of a neighborhood function $h_{R}$ that precisely defines the neighborhood borders (Eq. 8.5). Moreover, the computational effort needed to calculate the output-space distances from one DataBot to all others is reduced in such an approach because it is sufficient to look up radii coded in hash tables.

\subsubsection{Annealing Scheme}

The second problem with the SOP algorithm lies in the annealing scheme itself, which is not self-adaptive, as is claimed in [Herrmann, 2011]. This is because it is governed by two magic numbers: a threshold in terms of the number of DataBots that are allowed to jump and the maximum number of iterations after which an epoch ends given that this arbitrary threshold is exceeded in every iteration. The term "magic" indicates that these numbers are not derived from data but instead must be carefully chosen by an experienced user.

Only if the number of DataBots that want to jump exceeds a certain threshold value, called a fixed point in [Herrmann, 2011], will another iteration of the current epoch start. Otherwise, a new epoch with a smaller radius begins. This threshold value is required in SOP because the following case was not sufficiently considered: Often, as a result of a jump of one DataBot, not only will the scent of that DataBot change, but so will those of all the other DataBots in its new neighborhood and, more importantly, its old neighborhood. Because all DataBots are allowed to jump simultaneously, the DataBots are unable to update their scents sufficiently quickly in response to the changes occurring around them before they jump themselves; the scent at a possible new position is compared with an outdated (incorrect) scent at the current position, because the scent at the current position will have changed as a result of the jumps of other DataBots. This may result in random jumping.

In addition, if the scents at their current positions become worse, other DataBots will become unhappy. Therefore, on the one hand, they should also be allowed to jump, but on the other hand, allowing these DataBots to jump could trigger a cyclic process in which the DataBots simply follow each other. There is also a possibility that when DataBots are unhappy with their current positions, they may be unable to find new ones. Either no open positions may exist, or the scents at all other positions in the small circle around the DataBot itself may be even worse. This occurs because in a Gaussian distribution, there is a very high probability of making only small jumps and an exponentially lower probability of making larger jumps.

To summarize, these problems are intrinsic to the SOP algorithm and are unrelated to the sparse probabilistic movements of the agents, as claimed by [Herrmann, 2011, p. 66].

Another problem with the annealing process in SOP is the assumption that the stress $\mathrm{S}(\lambda, e)$ will be decreased only through iterations (Fig. 4.3 in [Herrmann, 2011, p. 69]) in which the DataBots move.

If the neighborhood function $F_{R}$ is chosen to be a Gaussian distribution, then a smaller radius implies a reduction of the neighborhood function, i.e., $R_{1}<R_{2}=>F_{R_{1}}<F_{R_{2}}$, because the standard deviation is defined by the radius. As shown by the curve in Fig. 4.3 in [Herrmann, 2011, p. 69], the sum of the scent ${ }^{64}$ in a neighborhood (in Hermann's thesis, this is called the

\footnotetext{
${ }^{64}$ Defined in chapter 7.1.
} 
sum of (topographic) stress) therefore also decreases because for lower values of the neighborhood function $F_{R}$, the scent ${ }^{64}$ values and, consequently, the stress $\mathrm{S}$ must be lower:

$F_{R_{1}}>F_{R_{2}}=>\lambda\left(R_{1}\right)<\lambda\left(R_{2}\right)=>S\left(R_{1}\right)<S\left(R_{2}\right)$. Only if the iterations are within the same epoch (with a constant radius $R$ ) must a reduction in stress be driven by DataBot movement. Therefore, applying argmin between scent ${ }^{64}$ values associated with different neighborhood radii results in random jumping of the DataBots.

Furthermore, the annealing scheme appears to reduce the stress $\mathrm{S}$ until convergence is reached (see Fig. 4.3 in [Herrmann, 2011, p. 69]). However, defining the scent ${ }^{64}$ and $\operatorname{Rmin}=1$ for the SOP algorithm as proposed by Herrmann results in $\lambda=\infty$ if there are no other DataBots in the neighborhood of a jumping DataBot. Even worse, this could lead to random jumping if, for example, two simultaneously jumping DataBots can smell only themselves when changing positions or if a reduction in the scent is only an effect of a reduction in the number of DataBots in the neighborhood.

By contrast, in Eq. 8.6 the payoff $\lambda_{e}\left(b_{j}, R_{e}\right)$ considered in Pswarm was modified based on symmetry considerations, because the two-dimensional output-space distances are irrelevant if the coordinate system is polar. In this case, it is sufficient simply to use radii, and thus, it is not necessary to simulate radial neighborhoods by means of expensive computations using a Gaussian neighborhood function. Pswarm allows the definition of a sharp, radial, and deterministic neighborhood function (called Cone, Eq. 8.5) instead of the blurry, squarer than radial, and stochastic neighborhood of SOP.

In Pswarm, the "fixed point condition" of [Herrmann, 2011] is replaced with the equilibrium of happiness, $\frac{\partial S}{\partial e}=0$ in Eq. 8.8. The use of the derivative makes it possible, during an epoch with a specific radius $\mathrm{R}$, to find an iteration in which changes to the positions of some unhappy DataBots will not change the global happiness of all DataBots. In other words, an unhappy DataBot may jump to a new, more profitable position to become happier, but the DataBots surrounding its old position will simultaneously be left with less profitable positions and, in turn, become unhappier. This results in a kind of equilibrium in which, on the global scale of the toroidal plane ${ }^{65}$, the DataBots are incapable of finding more profitable positions.

When the DataBots are not allowed to jump simultaneously, they are able to detect the payoffs related to other DataBots in their current positions before deciding to jump. By allowing all DataBots to jump in every iteration, as in SOP, the process of finding emergent structures could be delayed or even destroyed.

On a toroidal grid, setting the maximal neighborhood radius to the maximal distance on the grid results in self-interaction of the DataBots: the probabilities of choosing a new position will overlap for radii that extend beyond the closer edge of the grid ( $R>$ Lines $/ 2$ if Lines $<$ Columns $)$. Moreover, the neighborhood of one DataBot will overlap with itself, which will result in an incorrect calculation of the payoff and disrupt the process of emergence. Furthermore, the (maximal) neighborhood radius $\mathrm{R}$ in SOP is determined based on the architecture of the latticeshaped output space [Herrmann, 2011, p. 138], which was set to a constant value of 64x64 in the cited thesis regardless of the specific structures of the various data sets to be analyzed.

\footnotetext{
${ }^{65}$ This statement is only true if the possible jump length does not decrease with the neighborhood size.
} 
Using Schelling's model in SOP is difficult because the dependence on chance, the data and the parameter settings causes an enormous number of iterations to be required [Hatna/Benenson, 2012] for the separation of the DataBots. Consequently, the number of iterations must be limited, and a threshold must be set on the number of jumping DataBots. Additionally, the attempt to find the minimum scent between two possible positions results in the problems discussed above. By contrast, Pswarm exploits the Nash equilibrium concept [Nash, 1950] based on the redefinition of scent as a payoff function $\lambda_{e}$ and important changes to the neighborhood definition. This results in an annealing scheme that is based on the data.

In conclusion, SOP requires the user to choose a lattice size, two magic numbers for the annealing process and, in some cases, a minimal radius, whereas Pswarm does not. Additionally, the annealing scheme of Pswarm is fully radial with sound neighborhoods, whereas the neighborhood definition and annealing process of SOP are inconsistent with each other, which could prevent effective self-organization and, thus, emergence (examples in chapter 10.3).

\subsubsection{Swarm Intelligence and Self-Organization}

As described in the previous chapter, swarm behavior is characterized by five main principles [Grosan et al., 2006]: Homogeneity, Locality, Collision Avoidance, Velocity Matching and Flock Centering. In Pswarm, every agent is based on a DataBot, and the motion of each DataBot is influenced only by a well-defined neighborhood in which no two DataBots can be located in the same place at the same time. Hence, the first three main principles are obviously used. Velocity is defined as the rate of change in position with respect to time.

Considering fluctuations due to randomness, the average change in position is defined as $\Delta \bar{R}=\frac{1}{2}\left|0.5-\frac{\text { Lines }}{2}\right|=\frac{\text { Lines }-1}{4}$ because the DataBots can jump with uniform probability to positions at distances ranging from 0.5 to $\frac{\text { Lines }}{2}$ units of length and the relevant time interval is one iteration (within an epoch). Therefore, on average, the agents in Pswarm exhibit velocity matching. Flock centering, in our case, refers to centering around more than one flock, if a flock is understood to have the figurative meaning of a group of similar agents. In summary, all five principles of swarm behavior are represented in Pswarm. For the simplified definition of intelligence reduced to behavior, as presented in the last chapter, Pswarm therefore uses swarm intelligence.

Self-organization relies on four principles [Bonabeau et al., 1999]: positive and negative feedback, amplification offluctuations and multiple interactions. Fluctuations appear because of the random jump lengths and the random choices of new DataBot positions. Multiple interactions among DataBots are required for stigmergy in a given neighborhood in which various DataBots are present. Positive feedback and negative feedback are reflected in the choices of a DataBot to not jump when it is "happy" and to jump when it is "unhappy". Moreover, the number of DataBots cannot be reduced because each DataBot represents one data point in the data set. Consequently, self-organization is a property of Pswarm if the data set of interest contains more than 100 high-dimensional data points. Because of the randomness of the choice of possible jump positions, the system is temporally and structurally unpredictable, and Pswarm exhibits multiple interactions among many agents. The property of irreducibility is shown through the found compact and connected structures (chapter 10-12). Therefore, this system of DataBots possesses the property of emergence, as defined in chapter 7.3. 


\subsection{Clustering on a Generalized U*-Matrix}

Chapter 4 introduces a generalized $U^{*}$-matrix visualization called topographic map that can be used for any projection method. The $\mathrm{U}^{*}$-matrix represents high-dimensional density- and distance-based structures and is visualized as a topographic map with hypsometric tints [Thrun et al., 2016a]. Chapter 4 explains the connection between an approximation made by the simplified ESOM (sESOM) algorithm and an abstract U-matrix (AU-matrix) [Lötsch/Ultsch, 2014]. The clustering approach here uses the idea applied for the ESOM method that the abstract $\mathrm{U}^{*}$ matrix can be used for hierarchical clustering [Ultsch et al., 2016a].

Here, Pswarm, the AU-matrix concept and the proposed visualization are combined in the DBS clustering approach. In contrast to SOP and ESOM, this semi-interactive approach does not require any parameters other than the number of clusters and the cluster structure, which is either connected or compact (for details, see chapter 3). The number of clusters and the cluster structure can be estimated by counting the valleys in a topographic map and from a dendrogram. If the number of clusters and the clustering method are chosen correctly, then the clusters will be well separated by mountains in the visualization. Outliers are represented as volcanoes and can be interactively marked in the visualization after the automated clustering process.

The distances required for hierarchical clustering are defined by the AU-matrix, which was introduced in [Lötsch/Ultsch, 2014] for the U-matrix of a SOM. Here, the AU-matrix itself is defined by the Pswarm projection. In principle, the approach described in this section can be used for clustering based on any projection method because it is possible to generate a generalized U-matrix for any projection method (see chapter 5).

Let $\mathrm{G}(\mathrm{l}, \mathrm{j}, \mathcal{D})$ be the minimum of all possible path distances $\mathrm{p}_{\mathrm{j}, 1}$ between a pair of points $\{j, 1\} \in$ $O$ in the output space, as defined in chapter 2; then, the graph $\mathcal{D}$ is defined as the Delaunay graph weighted by the high-dimensional Euclidean distances between the points $\{j, 1\} \in \mathrm{I}$ in the input space. In every direct neighborhood $H_{j}(k=1, \mathcal{D}, O)$, all direct connections from the points $l$ to the point $j$ in the output space are weighted using the input-space distances $\mathrm{D}(1, \mathrm{j})$. In comparison to the ESOM clustering method proposed in [Ultsch et al., 2016a], here the shortest paths $G(l, j, \mathcal{D})$ are calculated additionally using the algorithm of [Dijkstra, 1959]. Contrary to [Ultsch et al., 2016a], the DBS clustering is not based on density information coded in the Pmatrix, because Pswarm itself is already able to project density-based structures (e.g. projection of EngyTime in chapter 10.3, Figure 10.7).

For example, in Figure 8.3, there are two well-separated clusters (green and blue), which the compact DBS clustering can detect in the dendrogram (Figure 8.4, left). In fact, the dendrogram could indicate also three or four clusters, but this is not verified by the visualization. If three or four clusters were chosen, the DBS clustering algorithm would not label points in the same cluster with the same color because they would not be well separated by mountains. The cluster heatmap shown in Figure 8.4 (right) verifies the clustering result of two clusters.

The outliers in a data set may be manually identified by the user. In this case, choosing the connected structure option for the clustering process would result in the automatic detection of all outliers. However, this option does not always lead to the detection of the main clusters in terms of the $G(l, j, \mathcal{D})$ distances. A second example of outlier detection is presented in chapter 10 using the Tetragonula data set [Franck et al., 2004]. 


$$
\text { 产 }
$$

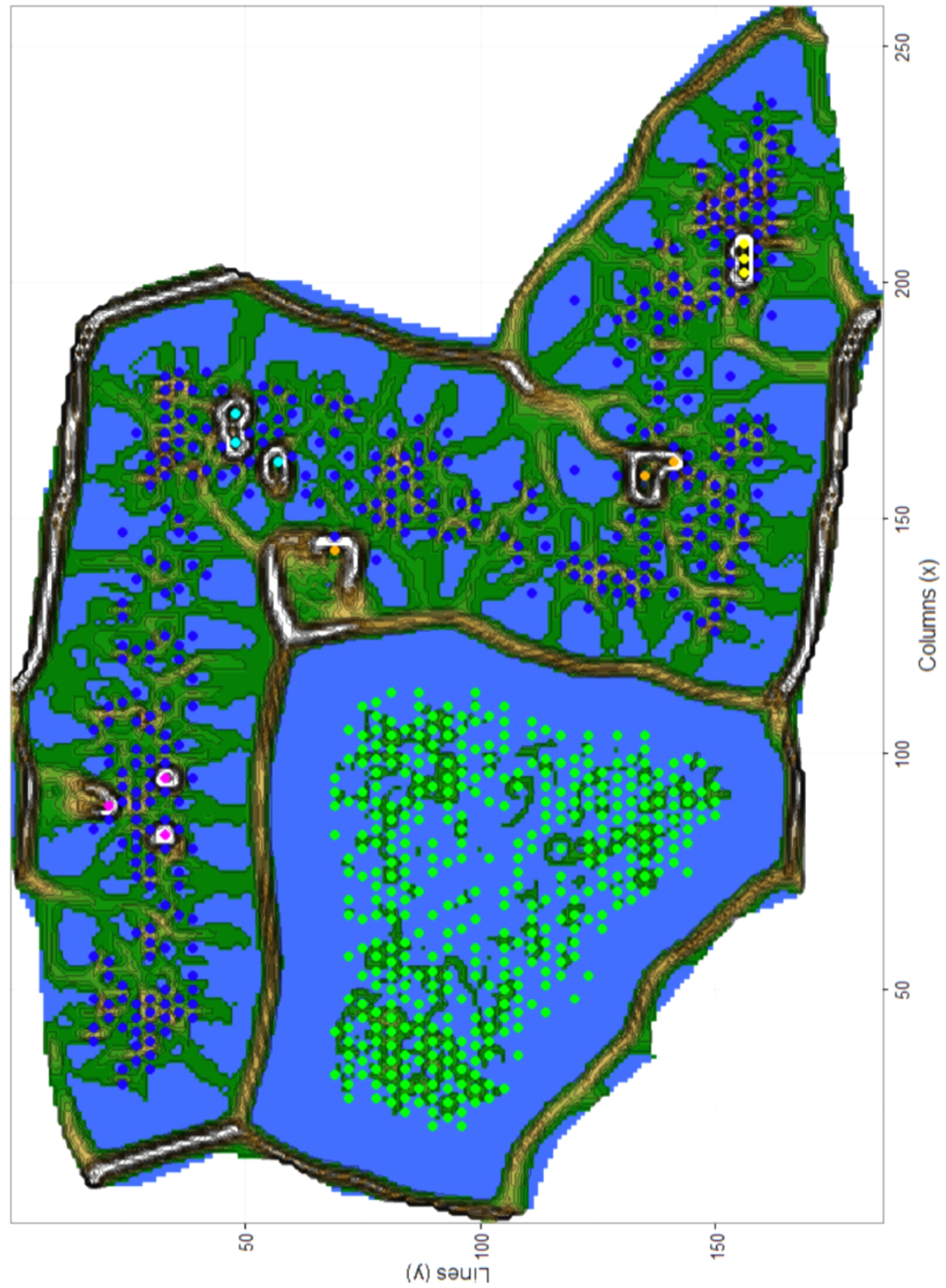

Figure 8.3: DBS visualization as a topographic map of the Target data set of [Ultsch, 2005a]. Two main clusters are shown; the cluster labeled in green has a higher density than the cluster labeled in blue. The outliers (orange, yellow, magenta and cyan) lie in volcanoes. 

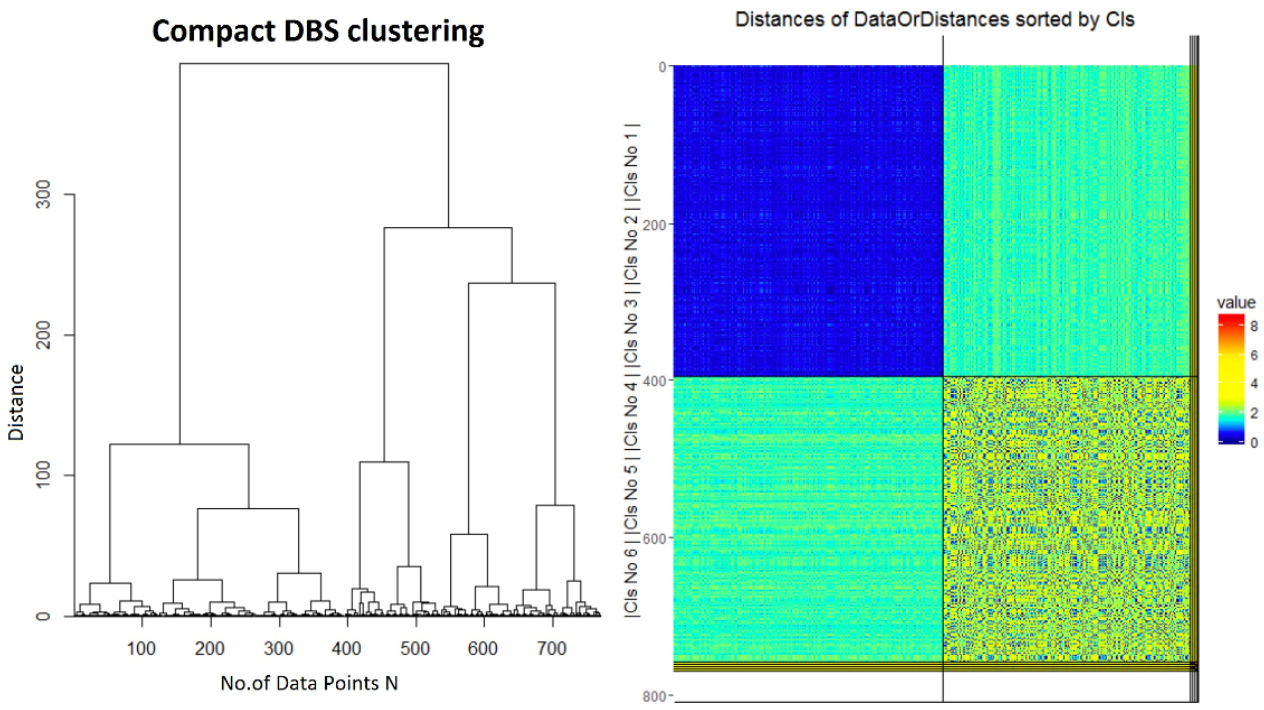

Figure 8.4: The dendrogram (left) of Target data set generated using the Ward algorithm shows either two or four clusters; however, in Figure 8.3, only two clusters are visible. The heatmap of the Target data set (right) shows two separated clusters with some outliers, because the intracluster distances are distinctively smaller than the intercluster distances.

Open Access This chapter is licensed under the terms of the Creative Commons Attribution 4.0 International License (http://creativecommons.org/licenses/by/4.0/), which permits use, sharing, adaptation, distribution and reproduction in any medium or format, as long as you give appropriate credit to the original author(s) and the source, provide a link to the Creative Commons license and indicate if changes were made.

The images or other third party material in this chapter are included in the chapter's Creative Commons license, unless indicated otherwise in a credit line to the material. If material is not included in the chapter's Creative Commons license and your intended use is not permitted by statutory regulation or exceeds the permitted use, you will need to obtain permission directly from the copyright holder. 